\title{
ETIENNE ACHILLE et LYDIE MOUDILENO, Mythologies postcoloniales. Pour une décolonisation du quotidien
}

\section{Alexandre Calvanese}

\section{(2) OpenEdition}

\section{Journals}

\section{Édition électronique}

URL : http://journals.openedition.org/studifrancesi/16790

DOI : $10.4000 /$ studifrancesi. 16790

ISSN : 2427-5856

\section{Éditeur}

Rosenberg \& Sellier

\section{Édition imprimée}

Date de publication : 1 juillet 2019

Pagination : 200-201

ISSN : 0039-2944

\section{Référence électronique}

Alexandre Calvanese, « ETIENNE ACHILle et Lydie moudileno, Mythologies postcoloniales. Pour une

décolonisation du quotidien", Studi Francesi [En ligne], 187 (LXIII | I) | 2019, mis en ligne le 01 juillet 2019, consulté le 25 janvier 2021. URL : http://journals.openedition.org/studifrancesi/16790 ; DOI : https://doi.org/10.4000/studifrancesi.16790

Ce document a été généré automatiquement le 25 janvier 2021.

\section{(c) (i) (9) $\Theta$}

Studi Francesi è distribuita con Licenza Creative Commons Attribuzione - Non commerciale - Non opere derivate 4.0 Internazionale. 


\title{
ETIENNE ACHILLE et LYDIE MOUDILENO, Mythologies postcoloniales. Pour une décolonisation du quotidien
}

\author{
Alexandre Calvanese
}

\section{RÉFÉRENCE}

ETIENNE ACHILle et Lydie Moudileno, Mythologies postcoloniales. Pour une décolonisation du quotidien, Paris, Champion, 2018, 149 pp.

Quel est le rapport entre le journal de 13 heures de la chaîne TF1 et une comédie capable de grimper le hit-parade des entrées au cinéma, ou encore entre la couverture médiatique des réactions aux attentats de janvier 2015 à Paris et le rôle public de premier plan assumé par un ancien champion du monde de football de 1998? D'après les deux auteurs de ce livre, Etienne ACHILLE et Lydie MOUDILENo, il s'agit de multiples aspects dans lesquels se manifeste, de manière parfois inattendue, le «caractère racialisé d'une quotidienneté française censée être aveugle à la race» (p. 8). Cet aveuglement - «l'impensé de la race» selon les mots d'Achille Mbembe - consisterait dans l'incapacité de reconnaître et d'admettre la force opératoire de la notion de race, c'est-à-dire d'une catégorie de l'imaginaire historiquement et socialement construite, qui participe à la production d'un discours sur la race comme concept scientifique (employé pour différencier les êtres humains) même si la validité et la pertinence de ce dernier ont été depuis longtemps mises en discussion. Nier la fonction jouée au quotidien par cette construction de l'imaginaire produirait, selon les auteurs, un effet bien plus vaste, car cela reviendrait «à occulter la postcolonialité de la France, et avec elle toute une histoire coloniale absolument déterminante pour la saisie des identités contemporaines» (p. 9), mais aussi à méconnaître le racisme différentialiste qui a pris pied au cours de ces dernières décennies par le biais de signifiants moins dérangeants et plus acceptables comme, par exemple, la culture, l'ethnie, la communauté. C'est pour 
cette raison qu'Achille et Moudileno affirment la nécessité de penser la permanence du concept de race dans sa visibilité aussi bien que dans son invisibilité, et d'en dégager les occurrences dans le milieu où elle s'est déployée historiquement, c'est-à-dire l'imaginaire de la quotidienneté. C'est en ce sens que leur livre œuvre pour une décolonisation du quotidien, selon les mots du sous-titre.

2 La première partie du titre, en revanche, nous dit clairement le double héritage intellectuel dont se réclament les deux auteurs: d'un côté Roland Barthes et son livre de 1957, de l'autre la critique postcoloniale, représentée notamment par les travaux d'Edward Said et de Valentin Y. Mudimbe, sans oublier la leçon de Frantz Fanon. Si, en considération du sujet et de l'objectif de ce livre, ce dernier champ de références ne surprendra personne, on pourrait éprouver quelque étonnement face à sa contamination avec l'un des pères de la méthode structuraliste. Cependant - outre le fait que les auteurs reconnaissent à Mythologies le mérite d'offrir encore, soixante ans après sa parution, un outil critique très efficace pour lire le quotidien et «défricher le "champ privilégié des significations mythiques" de la France contemporaine» (p. 13) le livre de Barthes révélait déjà un intérêt aigu pour l'idéologie coloniale au moment même (le milieu des années cinquante) où celle-ci se trouvait confrontée au processus de la décolonisation. Le but déclaré par les deux auteurs est alors celui de rendre essentiel un aspect qui pour Barthes n'était qu'un des visages de l'idéologie bourgeoise, et de montrer que «la République [d'aujourd'hui] en crise d'identité est toujours productrice et preneuse de mythologies, et celles qui se rapportent à l'histoire coloniale ne manquent pas» (p. 17), au point qu' «un travail de localisation des formes coloniales naturalisées dans la culture populaire [...] semble indispensable» pour solliciter une «prise de conscience collective» (p. 20).

3 Les sept chapitres qui suivent la riche introduction de l'ouvrage sont donc autant d'essais dans lesquels les auteurs retracent et mettent en évidence le processus de naturalisation des valeurs et de cristallisation du monde qui se trouve à l'origine de toute mythologie (dans l'acception barthésienne du mot). Le lecteur sera ainsi invité à s'interroger (pp. 23-35) sur la persistance dans l'environnement public (à travers la toponymie, les monuments, les écoles et les bâtiments publics baptisés en hommage), d'un panthéon qui évoque des hommes de la $\mathrm{III}^{\mathrm{e}}$ République dont les noms sont étroitement associés à l'histoire du colonialisme et à l'idée de «la plus grande France», comme les Maréchaux Bugeaud, Lyautey, Gallieni et Joffre. Et, tout de suite après (pp. 37-47), à réfléchir sur la manière dont un rendez-vous quotidien pour des millions de français - le journal de 13 heures de TF1 - a pu élaborer un mythe apparemment inverse mais en réalité complémentaire, celui de «la plus petite France»: un récit nourri de nostalgie pour un pays censé être plus vrai et authentique, ancré dans les campagnes et dans les traditions rurales, et dont l'histoire est expurgée de toute trace coloniale (et bien sûr, ce repli «a-colonial» n'est pas anodin à une époque qui connaît, non seulement en France, de violentes réactions de rejet aux transformations socioculturelles du monde contemporain).

4 L'analyse des médias, et notamment des journaux télévisés, s'avère particulièrement fructueuse pour les deux auteurs, car ils y puisent un deuxième essai très mordant (pp. 79-94) dans lequel il est question de la non-représentation de la région antillaise et de ses habitants dans le cadre de la couverture médiatique des réactions aux attentats de janvier 2015 à Paris: une absence qui devient signifiante si l'on constate que les Antilles ne sont pas systématiquement absentes des représentations collectives faites par la 
Métropole (par exemple quand il s'agit des reportages sur le pique-nique aux Antilles du 2 janvier), et qui doit donc être lue comme l'autre volet du double mythe élaboré par la France en relation à cette région: d'un côté le rêve exotique, de l'autre l'affirmation, en large partie conséquence de la désintégration de l'empire colonial, de l'hexagonalité de la France. Selon les circonstances, donc, le territoire juridique de la nation peut céder la place à «une carte amputée de son outre-mer» (p. 93). Carte qui peut toutefois - car les contradictions sont à l'ordre du jour dans les mythologies quotidiennes, et les deux auteurs ne perdent pas l'occasion de les relever - reprendre ses limites originelles en relation aux enjeux culturels et géopolitiques du moment: voici alors que la figure du «Grantécrivain noir» (pp.111-125) permet de redonner de la sève à un mythe national (celui du grand écrivain français) qui semblait s'être épuisé après les années soixante-dix, et de dépister les diagnostics de déclin culturel et linguistique dont la France semble souffrir depuis quelque temps. Emblématique de cette attitude ambivalente a été l'hommage rendu à Aimé Césaire après sa mort, dans lequel toute référence à la dimension contestataire du poète de la Négritude a été stratégiquement effacée pour en faire un "grand écrivain» tout court de la République. En même temps, nous disent Achille et Moudileno avec une pointe polémique non dissimulée, cette politique ne serait pas possible sans la disponibilité de certains "granzécrivains noirs» (Dany Laferrière et Alain Mabanckou méritent une mention spéciale) qui, finalement, se prêtent à nourrir le mythe d'une diversité et d'une intégration accomplies «sans heurts, dans la jovialité et les références partagées» (p. 125). Attitude qui finit par réaffirmer le rôle de Paris, sa centralité dans le monde, son pouvoir d'octroyer des honneurs et de valider des itinéraires artistiques. 